\title{
Simulations of Transport and RF Power Production in a 35-GHz Relativistic Klystron*
}

\author{
S.M. Lidia, LBNL, Berkeley, CA 94720 USA \\ J. Gardelle, T. Lefevre, J.L. Rullier, CEA/CESTA, BP 2, 33114 Le Barp, France \\ J.T. Donohue, CENBG, BP 120, 33175 Gradignan, France \\ P. Gouard, CEA/CIF, BP 12, 91680 Bruyeres-le-Chatel, France
}

\begin{abstract}
A current experiment is underway to study beam dynamics and rf production in a $35-\mathrm{GHz}$ relativistic klystron using a 1-kA, 7-MeV electron beam produced by the PIVAIR accelerator. We present simulations of transport in the PIVAIR accelerator, modulation from a free-electron laser interaction, post-wiggler solenoidal transport, and interaction with two rf cavities. These simulations are performed with a suite of various codes. Steady-state and transient effects are discussed. The calculated transverse and longitudinal profiles of the beam are compared with experimental diagnostic measurements.
\end{abstract}

\section{INTRODUCTION}

An experiment to produce a $35 \mathrm{GHz}$ modulated beam by a FEL, and to subsequently extract power from this beam in a resonant cavity has been performed at CESTA. This work is in support of the study of two beam accelerators based on relativistic klystrons, and has been executed jointly by groups at LBNL, CEA/CESTA, and CERN. One of the aims of this current experiment is to validate and benchmark our simulation codes.

For the design of the experiment and the analysis of its results, we have relied upon four distinct codes: an envelope code for basic transport studies; PARMELA [1] for more detailed examination of the transverse phase space evolution; SOLITUDE [2] for the study of 3D, steady-state FEL interactions; and RKS [3] to study the time-dependent, 3D bunch evolution and power production in output microwave cavities. A brief description of the experiment is given elsewhere in these proceedings [4].

\section{TRANSPORT IN PIVAIR}

The simulation begins with a description of the transport from the cathode to the experiment. For this the envelope code is solely used. A plot of the magnetic field and beam edge radius is given in Figure 1.

The currents in the two final solenoids and dipole coils are adjusted for proper matching into the wiggler. A Rogowski coil placed at the entrance to the wiggler measured the beam current; $800 \mathrm{~A}$ were obtained in the current experiment.

\footnotetext{
${ }^{*}$ Work performed at LBNL was supported under the auspices of the
} U.S. Department of Energy under Contract No. AC03-76SF00098.

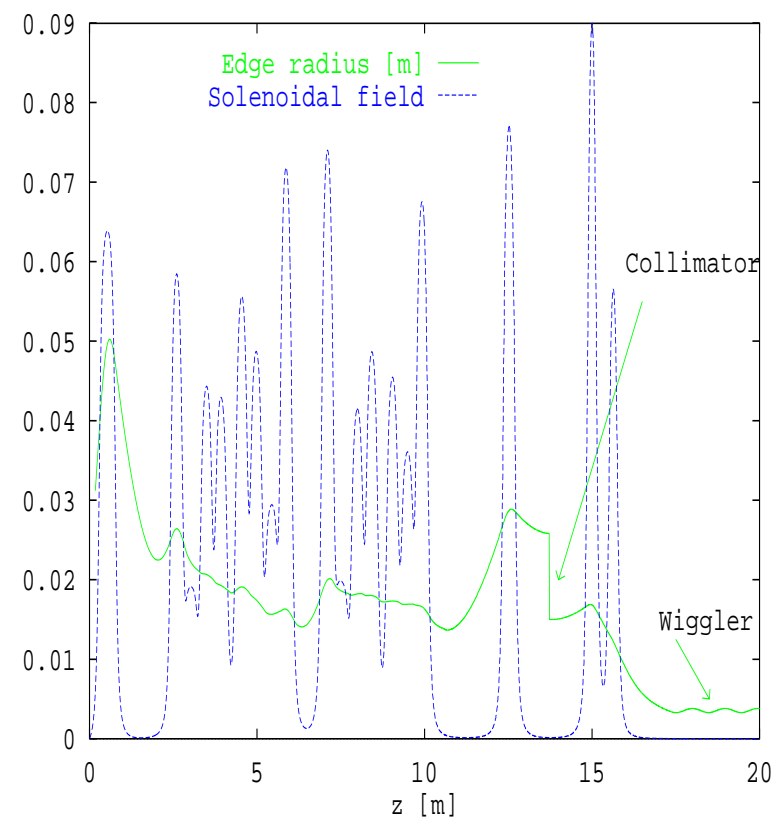

Figure 1: Transport in PIVAIR.

\begin{tabular}{|c|c|}
\hline Wiggler period & $20 \mathrm{~cm}$ \\
\hline \hline Resonant field & $1650 \mathrm{G}$ \\
\hline Total no. periods & 32 \\
\hline Total length & $6.4 \mathrm{~m}$ \\
\hline Input signal frequency & $35.04 \mathrm{GHz}$ \\
\hline Input signal power & $5 \mathrm{~kW}$ \\
\hline DC current at entry & $1000 \mathrm{~A}$ \\
\hline
\end{tabular}

Table 1: FEL parameters.

\section{TRANSPORT THROUGH THE FEL}

The main FEL parameters are listed in Table 1. SOLITUDE has been run to obtain fair agreement with the preliminary results of the current experiment, ie. $40 \mathrm{MW}$ of FEL power and $250 \mathrm{~A}$ of current at the wiggler exit. Besides the basic parameters of the beam distribution (current, radius, emittance) and the input microwave power, SOLITUDE has a few additional 'knobs' with which to tune the resultant interaction. These allow the simulation to place the entry of the beam centroid at any radius and polar angle in the entry plane, and with two independent angles with respect to the normal of the entry plane. Sensitivity studies have shown that for small variations from normal, 


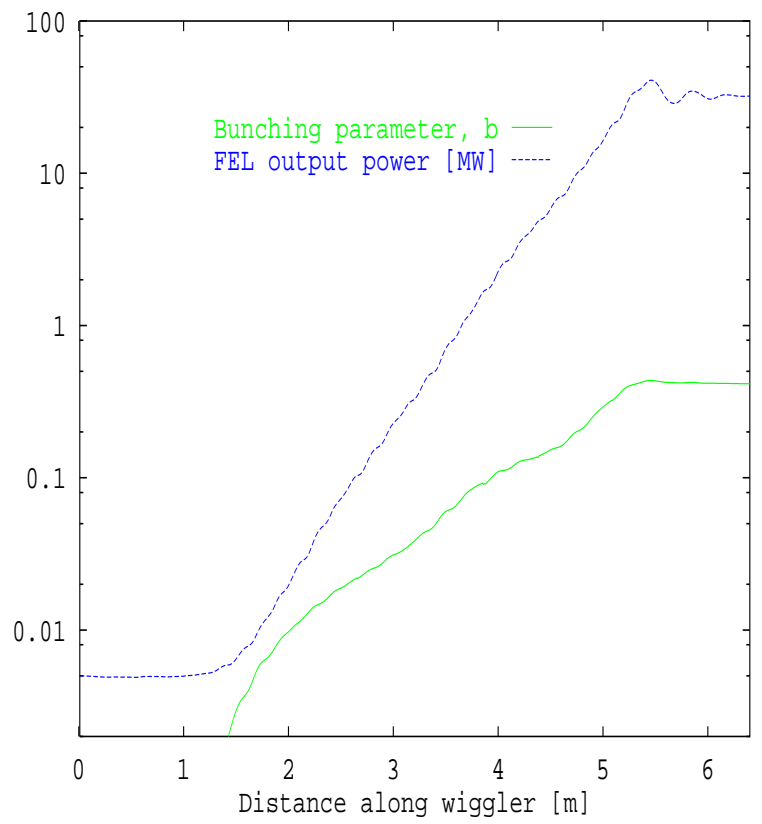

Figure 2: Evolution of FEL power and bunching.

on-axis injection, it is the azimuthal angle (angular divergence from the wiggler axis) of the centroid motion which has the largest effect on overall FEL performance. In particular, we have found that an azimuthal angle of $\sim 33 \mathrm{mrad}$ at the entrance plane generates trajectories within the FEL region that lead to the observed $40 \mathrm{MW}$ of output power and $250 \mathrm{Amps}$ of current at the exit current monitor. The bunching parameter at the FEL exit is given by SOLITUDE as $b \sim 0.4$. The evolution of the output power and of the bunching parameter as calculated by SOLITUDE is shown in Figure 2. The output data generated by SOLITUDE include the detailed particle distribution in the horizontal and vertical phase spaces, as well as the distribution in particle energy and arrival time for a given bunch. These data provide an indication of the initial conditions for simulations of the downstream region.

\section{DOWNSTREAM TRANSPORT AND CAVITY INTERACTION}

After a short drift space, the bunched beam is focused by a solenoid module (peak axial field $~ 0.27 \mathrm{~T}$ ) into one of two Ka-band standing-wave RF output cavities. These cavities were designed using standard electromagnetic codes, and were built by the CLIC group at CERN. The measured parameters ${ }^{1}$ of these cavities are shown in Table 3 .

The transport from the end of the wiggler through the cavities has been studied with PARMELA, the envelope code, and RKS. The initial conditions generated by SOLITUDE have been used in the envelope simulation. In the case of the RKS code, on the other hand, we have slightly

\footnotetext{
${ }^{1}$ These shunt impedance values have not been directly measured, but have been inferred from the beam interaction and the measured power.
}

\begin{tabular}{|c|c|c|}
\hline Resonant frequency $[\mathrm{GHz}]$ & 35.0 & 35.6 \\
\hline \hline Quality factor, $Q_{\text {Loaded }}$ & 60 & 271 \\
\hline Shunt impedance [R/Q] $[\Omega]$ & 76. & 76. \\
\hline DC current in cavity [A] & 120 & 120 \\
\hline Measured output power [MW] & 12 & 0.69 \\
\hline
\end{tabular}

Table 2: RF cavity parameters.

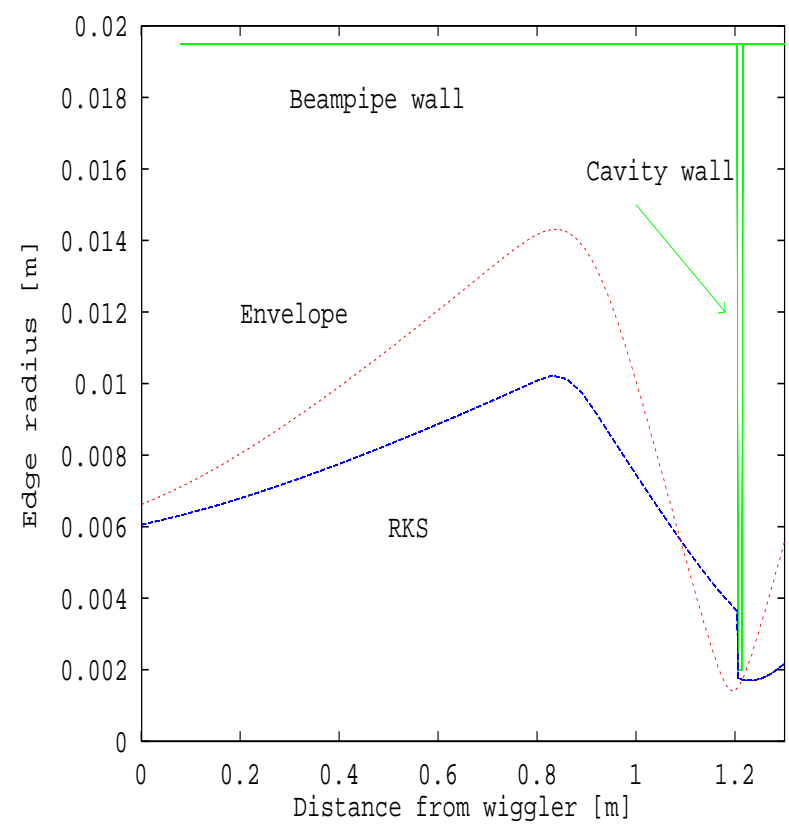

Figure 3: Beam edge radius evolution.

adjusted the initial conditions to obtain fair agreement with the experimentally measured beam spot sizes, and the incident and transmitted beam currents through the cavities. The trajectories calculated by the envelope code and RKS are shown in Figure 3.

The differences between the two codes are an indication of the effects of the detailed statistics of the transverse dynamics. The envelope code assumes an on-axis beam described by a transverse KV and a longitudinally uniform distribution, while the RKS code uses a semi-Gaussian distribution for the transverse phase space and a Gaussian distribution for the longitudinal phase space. The 'edge' radius in this case is twice the rms radius. These different models present slightly different modes of evolution, while showing fair agreement on the measured parameters at the end points. RKS reproduces good agreement with experiment in predicting a $4 \mathrm{~mm}$ spot size radius at the cavity plane.

Measurements of the FEL output power give an indication of the bunching parameter of the beam at the FEL exit. This can be measured experimentally [5]. Subsequent measurement of the cavity output power and beam current allows us to indirectly measure the cavity shunt impedances. The steady-state output power from the cavities follows 
Proceedings of the 1999 Particle Accelerator Conference, New York, 1999
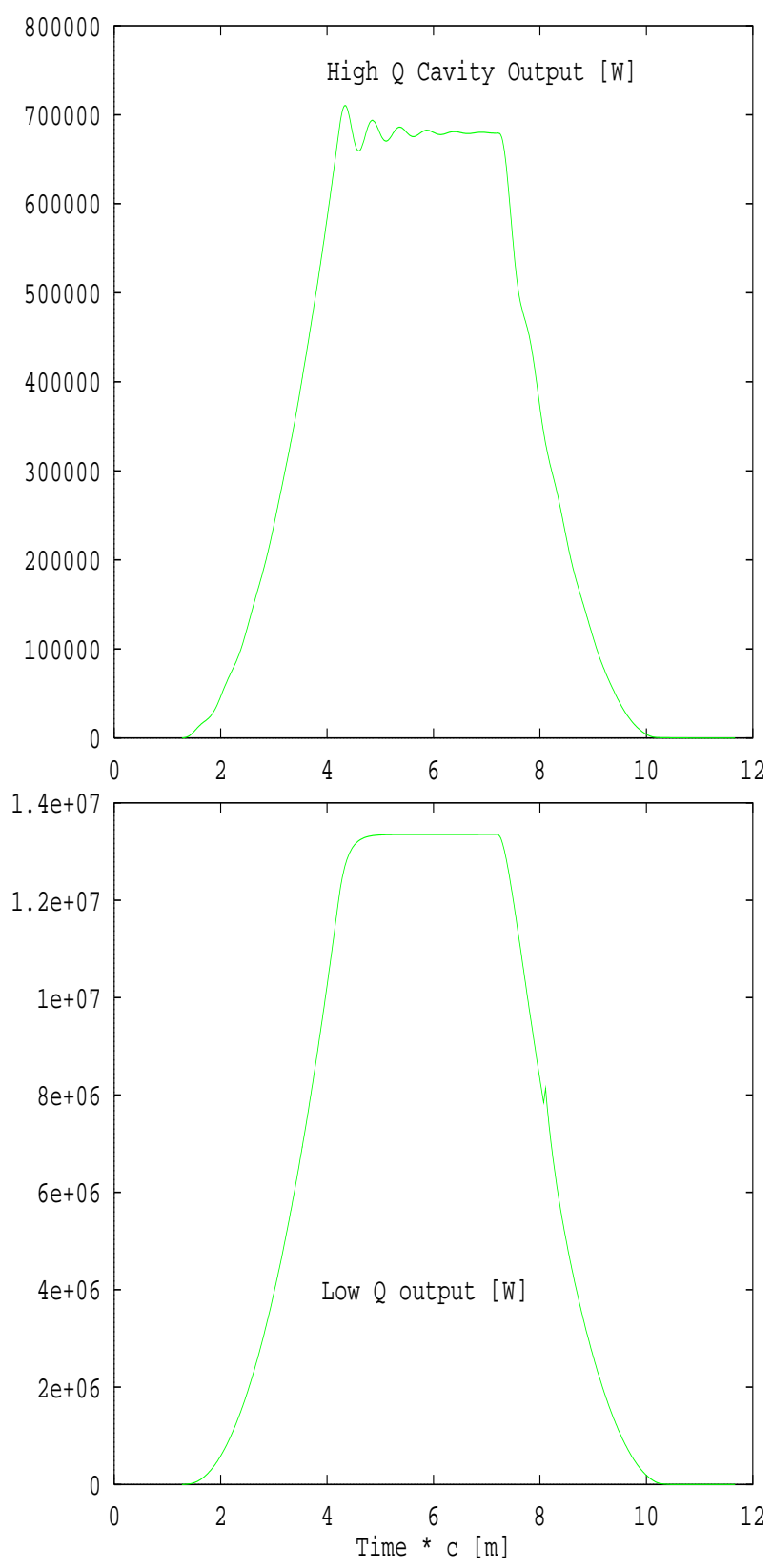

[1] Parmela. Maintained by the Los Alamos Accelerator Code Group.

[2] J. Gardelle et. al. Free-electron laser simulations: Effects of beam quality and space charge. Phys. Rev. E, 50:4973, 1994.

[3] S. Lidia. Relativistic klystron simulator. Unpublished, 1999.

[4] T. Lefevre et. al. Results on the interaction of an intense bunched electron beam with resonant cavities at $35 \mathrm{ghz}$. In these proceedings, 1999.

[5] J. Gardelle et. al. Direct observation of beam bunching produced by a high power microwave free electron laser. Phys. Rev. E, 79:3905, 1997.

[6] J.C. Slater. "Microwave Electronics". D. Van Nostrand Company, Inc., 1950.

[7] S. Lidia. Single-mode beam-cavity interaction in relativistic klystrons. In these proceedings, 1999.

Figure 4: Temporal power development in the two cavities.

from the relation

$$
P_{\text {out }}=I_{d e}^{2} b^{2} Q_{\text {Loaded }}\left[\frac{R}{Q}\right] \cos ^{2} \psi
$$

where $b$ is the bunching parameter, and $\psi$ is the cavity tuning angle [6]. The temporal evolution of the output cavity power is calculated by the RKS code. The output from the low- and high-Q cavities is shown in Figure 4. The temporal evolution of the power and phase in the output cavities is calculated by an equivalent circuit equation coupled to the detailed beam dynamics [7]. 